\title{
L'augmentation du bénéfice social grâce à la chirurgie bariatrique
}

\author{
Jean-Marie Mégevanda, Jean-Marc Heinicke ${ }^{b}$, François Pralong ${ }^{c}$ \\ a Dr, Médecin-chef, service de chirurgie viscérale, Hôpital de la Tour; ${ }^{b}$ Dr, Médecin-chef, service de chirurgie viscérale, Hôpital de la Tour; \\ ${ }^{c} \mathrm{Pr}$, Médecin-chef, Centre d'endocrinologie, diabétologie et obésité, Hôpital de la Tour
}

La proportion des personnes obèses a presque doublé au cours des vingt dernières années, entraînant non seulement une réduction de l'espérance et de la qualité de vie des personnes concernées, mais aussi un impact économique de 8 milliards de francs. Les traitements directs (y compris les opérations) génèrent à peine $1 \% \mathrm{de}$ ces dépenses, tout en réduisant le principal facteur de coût - les comorbidités et en améliorant la vie des patients.

\section{Obésité: une souffrance chronique avec plusieurs comorbidités}

Madame S. s'est présentée à notre consultation pour la première fois il y a cinq ans, à l'âge de 38 ans, envoyée par son médecin de famille pour une prise en charge de son obésité. Lorsque nous l'avons rencontrée, elle avait dû reprendre son travail d'aide-soignante après un divorce six ans auparavant, et élevait seule ses deux enfants de douze et dix ans. Lors de ses grossesses, elle avait pris à chaque fois une quinzaine de kilos qu'elle n'avait pas réussi à perdre ensuite, et depuis son divorce, son poids s'était remis à augmenter, pour atteindre les 120 kilos qu'elle pesait lors de la première consultation. Elle avait déjà fait des régimes, avec une anamnèse de yoyo pondéral à deux reprises, perdant à chaque fois une dizaine de kilos, les reprenant ensuite rapidement. Son poids commençait à la gêner dans son travail et sa vie de tous les jours, et elle souffrait d'une hypertension artérielle traitée, d'un syndrome des apnées du sommeil traité par une assistance ventilatoire nocturne, ainsi que d'un pré-diabète sans traitement.

\section{L’obésité: plus qu'un défi individuel}

Les patients obèses posent des problèmes psychologiques et médicaux complexes, et pourtant l'obésité n'est reconnue comme une maladie à part entière par l'Organisation mondiale de la Santé que depuis 2006. De ce fait, l'enseignement spécifique concernant cette pathologie n'a été introduit que très récemment au sein de nos Facultés de médecine. Les complications associées à l'obésité, telles que le diabète, les problèmes respiratoires durant le sommeil, l'élévation du cholestérol, l'hypertension ou les douleurs articulaires ainsi que certains cancers, en font toute la gravité. Aider les patients à enrayer leur prise de poids, identifier les complications éventuellement présentes, puis les traiter sont autant d'actions qui nécessitent une expertise spécifique, et surtout l'intervention de différents professionnels de la santé: diététiciennes, psychologues, gastroentérologues, pneumologues, endocrinologues/ diabétologues, chirurgiens, médecins du sport et physiothérapeutes.

Cette approche interdisciplinaire demande certes des moyens supplémentaires. Elle constitue toutefois une des clés de la réussite du traitement, participant à remettre le patient au centre du réseau de soins et à le faire participer à la prise en charge de sa maladie et de ses conséquences néfastes. La trajectoire de vie de notre patiente illustre bien l'importance de cette prise en charge multimodale et globale à la fois. A défaut, on expose ces patients à la survenue d'épisodes de yoyo pondéral, à une prise de poids continue au fil des années s'accompagnant d'une diminution de leur qualité de vie ainsi que de leur espérance de vie.

L'objectif principal à long terme, particulièrement pour les jeunes patients, est la perte de poids. Les modifications du style de vie permettent des améliorations significatives du profil de risque cardio-métabolique et de la qualité de vie du patient, mais n'influencent finalement le poids que de manière modeste dans la vaste majorité des cas. Lorsqu'elle est applicable, la chirurgie de l'obésité, ou chirurgie bariatrique, est la 
seule approche thérapeutique permettant aux patients obèses morbides de perdre du poids de manière importante et durable.

Les données de santé publique disponibles en Suisse [1] confirment que cette approche est également bénéfique à l'économie. Les dépenses liées au traitement de

\section{La chirurgie bariatrique réduit significative- ment les dépenses liées au traitement des comorbidités et à leurs conséquences.}

l'obésité représentent environ $1 \%$ de la totalité des coûts engendrés par les maladies liées au surpoids et à l'obésité. De son côté, la chirurgie bariatrique permet de réduire significativement les dépenses liées au traitement des complications de l'obésité ou à leurs conséquences (baisse de productivité, absentéisme, invalidité), qui constituent $99 \%$ de ces coûts. De ce fait, cet argent est très bien investi.

\section{L'efficacité du by-pass gastrique}

En constant développement depuis le début des années 1990, ce type de chirurgie est clairement arrivé à maturité aujourd'hui: le profil de risque et de bénéfice, de même que le rapport entre coût et efficacité, sont clairement favorables à l'intervention. Ces différents indicateurs, dérivés de grandes études prospectives, parlent en faveur de l'approche chirurgicale, particulièrement pour des malades jeunes et présentant des complications métaboliques tels que notre patiente. Mme S. a bénéficié d'un by-pass gastrique un an après sa première consultation, après avoir suivi une préparation psychologique et nutritionnelle à l'intervention. Avant cela, elle avait dû démarrer un traitement antidiabétique oral. L'intervention a induit une perte d'environ 35 kilos durant la première année, accompagnée d'une normalisation de sa tension artérielle ainsi que de ses bilans glycémiques, permettant de retirer tous les médicaments. Elle n'a souffert que de peu d'inconvénients secondaires à la chirurgie: elle a présenté des malaises occasionnels apparaissant immédiatement après la prise des repas et qui sont bien maîtrisés par des adaptations alimentaires. En cela, Mme S. ressemble à la vaste majorité des personnes opérées qui perdent la plus grande partie de leur excès de poids après chirurgie bariatrique, ce qui s'accompagne d'une rémission à long terme des comorbidités associées. De ce fait, les patients peuvent arrêter la prise de nombreux médicaments, et bénéficient d'une réduction significative de leur risque cardio-vasculaire et de leur mortalité globale.

Cinq ans après son intervention, Mme $\mathrm{S}$. maintient un poids d'environ 90 kilos sans difficulté puisqu'elle a pu reprendre de l'activité physique régulière. Elle travaille à nouveau sans problème, son syndrome des apnées du sommeil a disparu, et elle peut également se passer de ses médicaments anti-hypertenseur et anti-diabétique. Elle a besoin de substitutions en fer, en calcium et en vitamine $\mathrm{D}$, prend également un comprimé multivitaminé par jour, et relève l'amélioration considérable que le by-pass gastrique a apportée à sa qualité de vie.

\section{Conclusion}

L'histoire de cette patiente est remarquable, car elle illustre bien les bénéfices engendrés par l'intervention chirurgicale et le suivi médical interdisciplinaire. Ces bénéfices se manifestent aussi bien dans l'amélioration de la santé et de la qualité de vie de la patiente, que d'un point de vue de santé publique. En effet, elle travaille à nouveau à plein temps. D'autre part, la disparition des complications permet de rétablir un profil de risque métabolique et cardio-vasculaire ainsi qu'une espérance de vie similaires à ceux de la population non obèse.

\section{Résumé}

Nous décrivons une patiente de 38 ans qui souffrait d'une obésité morbide, associée à plusieurs complications, rendant difficile la poursuite de son travail d'aide-soignante. Après une préparation adéquate, elle a bénéficié d'un by-pass gastrique qui lui a permis de perdre 35 kilos. Aujourd'hui, elle ne prend plus aucun médicament, travaille à nouveau à plein temps, et a retrouvé la qualité de vie qu'elle avait avant de prendre du poids: Non seulement la patiente se sent mieux, mais les coûts directs et indirects de son obésité morbide ont été réduits.

\footnotetext{
Référence

1 Office fédéral de la santé publique; Indicateur 5.2: Coûts du surpoids et de l'obésité (PDF, 101 kB, 19.6.2017).
} 